\title{
Glutathione-capped gold nanoclusters: photoinduced energy transfer and singlet oxygen generation ${ }^{\S}$
}

\author{
CHRISTIAN TALAVERA and PRASHANT V KAMAT*(D) \\ Radiation Laboratory, Department of Chemistry and Biochemistry, University of Notre Dame, Notre Dame, \\ IN 46556, USA \\ E-mail: pkamat@nd.edu
}

MS received 9 August 2018; revised 28 August 2018; accepted 31 August 2018; published online 27 September 2018

\begin{abstract}
Glutathione-capped gold clusters prepared in an aqueous medium are known to exhibit excellent photosensitizing properties. We have now successfully transferred these gold clusters in an organic medium while retaining all the characteristic excited state properties. These gold clusters can be further modified with organic ligands such as 2-Phenylethanethiol (PET). The gold clusters in organic medium exhibit enhanced emission yield $\left(\Phi_{\mathrm{f}}=0.15\right)$ compared to that in an aqueous medium $\left(\Phi_{\mathrm{f}}=0.08\right)$. The excited state lifetimes of $3.7 \mu \mathrm{s}$ (untreated) and $1.5 \mu \mathrm{s}$ (PET treated) in toluene are also greater than the lifetime observed in aqueous solution $(0.77 \mu \mathrm{s})$. By employing laser flash photolysis we are able to induce triplet energy transfer to $\beta$-carotene and oxygen. A singlet oxygen generation with the efficiency of $13 \%$ was observed in these experiments. The excited state properties of glutathione-capped gold clusters further shows its importance as a photosensitizer in light energy conversion and biomedical applications
\end{abstract}

Keywords. Gold clusters; singlet oxygen; excited state; transient absorption specroscopy; energy transfer; photosensitizer.

\section{Introduction}

Thiolated gold clusters have drawn significant interest because of their unusual photosensitizing properties. ${ }^{1,2}$ Compared to larger size gold nanoparticles, they do not possess plasmonic properties but exhibit long-lived excited states. ${ }^{3,4}$ The long-lived excited state of the gold cluster has an excited triple state character and it is attributed to the formation of the ligand-to-metal charge transfer (LMCT) state. These clusters which carry a metal core of few gold atoms and a shell of chemically bound thiols are known to exhibit molecularlike properties. ${ }^{5-7}$ These clusters have been found to be excellent photosensitizers in dye-sensitized solar cells and water splitting devices. ${ }^{8,9}$ By co-adsorbing gold clusters along with dyes such as Ru(III)trisbipyridyl complex and squaraine dye, we succeeded in boosting the open circuit potential of the dye-sensitized solar cell. ${ }^{10}$ Furthermore, the synergetic effects in enhancing the yield of excited states was also observed when these clusters were coupled with plasmonic silver and gold nanoparticles. ${ }^{11}$

In our previous study, we have investigated size dependent excited state transfer and charge properties of gold clusters. ${ }^{12}$ As the number of gold atoms increased from 10 to 25 , we observe a fast excited state decay component arising from the core atom transitions. Glutathione capped Au-clusters prepared in water usually have $10-16$ atoms and exhibit absorption in the visible $(<500 \mathrm{~nm}) .^{13}$

An interesting aspect of ligand exchange of nanoparticles is the ability to transfer the nanoclusters from polar to nonpolar medium using a phase transfer material. For example, tetraoctyl ammonium bromide (TOAB) has been widely used to prepare gold nanoparticles in toluene. ${ }^{14-16}$ The obvious question is whether

\footnotetext{
*For correspondence

$\S$ Dedicated to Professor M V George on the occasion of his $90^{\text {th }}$ Birth Anniversary.
}

Electronic supplementary material: The online version of this article (https://doi.org/10.1007/s12039-018-1549-6) contains supplementary material, which is available to authorized users. 
we can use this strategy to transfer thiolated gold clusters from the aqueous medium into an organic solvent and still retain the photosensitizing properties. Transfer of such clusters without significant alteration of the excited state can allow us to modulate their surface chemistry through additional ligand exchange. We now present here excited state properties of gold nanoclusters in toluene and discuss the energy transfer to molecular oxygen to produce singlet oxygen.

\section{Experimental}

The glutathione capped gold nanoclusters (AuNCs) were prepared using a modified procedure reported by Xie and co-workers. ${ }^{17}$ First, $0.24 \mathrm{~g}$ of gold(III) chloride trihydrate (99.9\%, Sigma-Aldrich) was dissolved in $100 \mathrm{~mL}$ of deionized (DI) water. In another flask, $0.27 \mathrm{~g}$ of L-glutathione (reduced, 98\%, Sigma-Aldrich) was dissolved in $100 \mathrm{~mL}$ of DI water. The two solutions were mixed together and an additional $100 \mathrm{~mL}$ of DI water was added to make up a total volume of $300 \mathrm{~mL}$. The mixture was stirred at room temperature for about $2 \mathrm{~h}$ until the solution turned from yellow to clear. After the solution turned clear, the mixture was placed in an oil bath at $70^{\circ} \mathrm{C}$ for $24 \mathrm{~h}$ with stirring. After $24 \mathrm{~h}$ elapsed, the solution turned from clear to dark-yellow and acetonitrile was added to precipitate the mixture of gold clusters. The precipitate was thoroughly washed with an acetonitrile and DI water mixture $(3: 1 \mathrm{v} / \mathrm{v})$ and finally redissolved in DI water. The gold clusters prepared by this method contain 10-12 gold atoms stabilized by glutathione. Detailed characterization can be found elsewhere. ${ }^{3,12}$

Phase transfer to toluene was carried out using a phase transfer agent tetraoctylammonium bromide (TOAB). The mixture of the two solutions upon shaking in a separating funnel allowed transfer of glutathione-protected gold clusters from water into toluene. In order to perform a ligand exchange with 2-phenylethanethiol (PET), the glutathione-protected gold clusters transferred in toluene solution with PET.

TOAB capped and PET capped AuNCs were further studied using steady-state and time-resolved optical measurements. UV-Vis spectra were recorded on Cary 50 Bio UV-Vis scanning spectrophotometer. Emission spectra were recorded using Horiba Jobin Yvon Fluorolog 3 spectrophotometer. Time-resolved optical measurements were carried out with nanosecond laser flash photolysis using a $337 \mathrm{~nm}$ Nitrogen laser for excitation. Other experimental details can be found in the Supplementary Information.

\section{Results and Discussion}

\subsection{Phase transfer of gold clusters}

Glutathione stabilized gold clusters prepared in aqueous solution are stable. Details on its preparation, characterization and excited state properties can be found elsewhere. ${ }^{3,12}$ The procedure of phase transfer (see Experimental section for details) involves treating the Au-Gltx clusters in aqueous solution with toluene containing tetraoctyl ammonium bromide (TOAB) solution in a separating funnel with a periodic shake. The TOAB encapsulates Au-Gltx clusters and facilitates its transfer to toluene. The cluster suspension in toluene can then be separated and any traces of water can be removed by treatment with anhydrous $\mathrm{Na}_{2} \mathrm{SO}_{4}$ crystals. This procedure is similar to the one adopted for transfer of gold nanoparticles from aqueous to toluene phase. ${ }^{14,15}$ The Au-Gltx clusters transferred in toluene are stable and are used for spectroscopic measurements. Additionally, a small batch was subjected to ligand exchange with 2-Phenylethanethiol (PET). The selection of PET was made to demonstrate the versatility of the gold capping with a simple aromatic thiol through ligand exchange. These phase transfer steps are pictorially represented in Figure 1 along with an illustrated scheme of phase transfer and ligand exchange with PET.

\subsection{Absorption and emission characteristics}

The absorption spectra of the TOAB capped and PET modified gold clusters in toluene were recorded along with those prepared in aqueous solution (Figure 2A). All these spectra exhibit similar absorption in the visible with the characteristic shoulder around $400 \mathrm{~nm}$. As shown earlier, the absorption of gold clusters is dependent on the size and shifts to red as we increase the size of the clusters. ${ }^{3,12}$ Based on the absorption characteristics we expect these clusters to contain 10-12 gold atoms.

The normalized emission and excitation spectra of three gold clusters are shown in Figure 2B. All three solutions were emissive with emission maximum around $605 \mathrm{~nm}$ region with little variation in the emission characteristics. A small blue shift $(2-5 \mathrm{~nm})$ in the emission band was noted for the clusters in toluene (PET treated and TOAB capped) as compared to AuGlx clusters in water. The excitation spectra of all three solutions matched the character of the absorption spectra and thus confirmed the emission to originate from the ligand to metal charge transfer complex transition. The similarity of the absorption and emission characteristics in aqueous and toluene solution indicates that the original Au-Glx core-shell structure obtained in the aqueous solution is retained following the phase transfer with TOAB and ligand exchange with PET in toluene. The TOAB or PET thus serves as an additional shell as it binds to Au-Glx cluster. This aspect of cluster rigidity in organic solvent further allows us to characterize the excited state properties. 


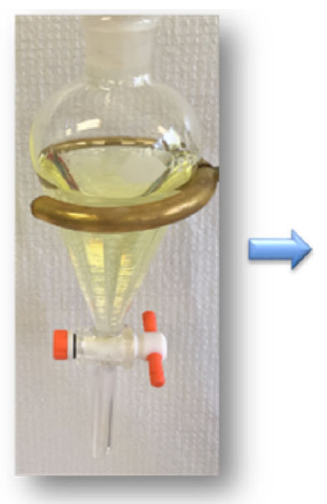

AuNCs in Water

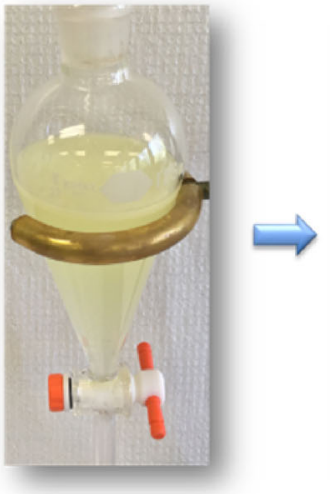

AuNCs in Water

+ TOAB/Toluene

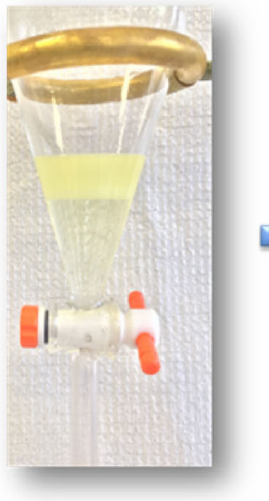

Phase transfer

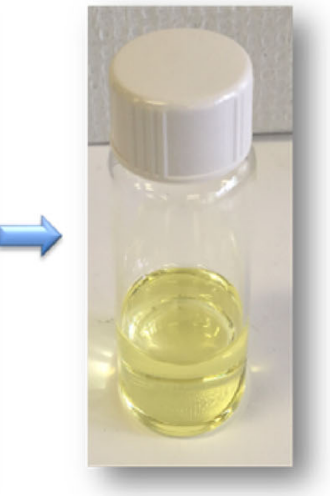

AuNCs in TOAB/Toluene
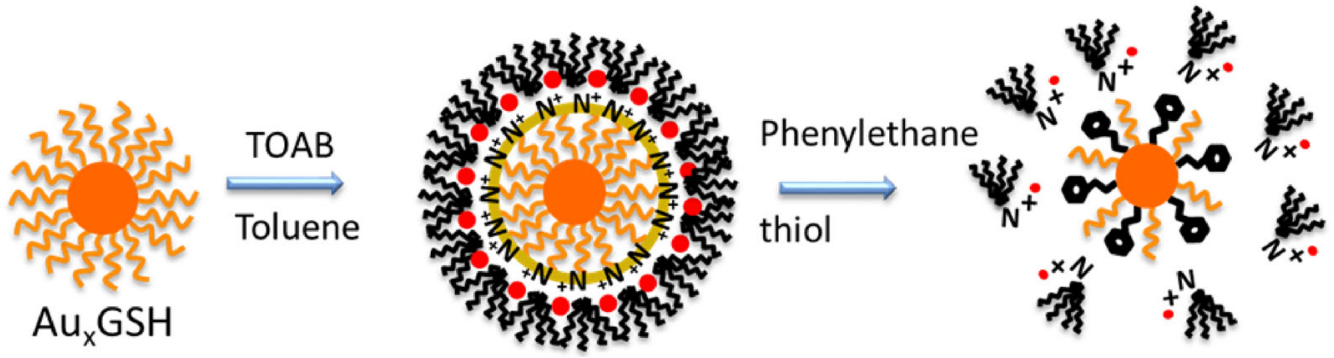

Figure 1. Top: Photographs showing the transfer of glutathione-capped gold clusters from aqueous solution into toluene with the aid of phase transfer agent, tetraoctyl bromide (TOAB). Bottom: Schematic illustration of the ligand exchange process.
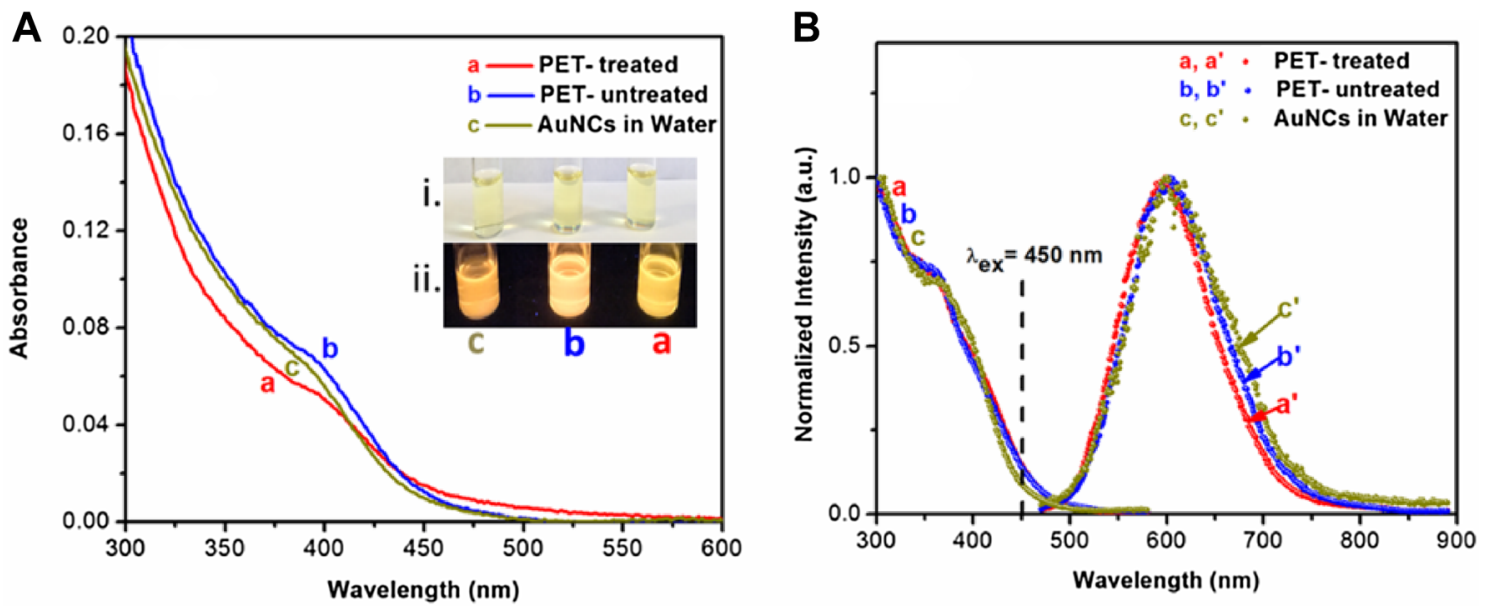

Figure 2. (A) Absorption spectra and (B) Emission (excitation wavelength $450 \mathrm{~nm}$ ) and excitation spectra (emission wavelength $620 \mathrm{~nm}$ ) of gold nanoclusters: a, a' PET treated (toluene); b, b' TOAB capped (toluene); and c, $\mathrm{c}^{\prime}$ in aqueous solution (as prepared). The photographs show the samples (i) under room light and (ii) under UV light.

Another feature of interest is the enhancement in the fluorescence quantum yield that we see when capped with TOAB or PET and suspended in toluene. The visual display of luminescence under UV light is presented in Figure 2A. Both PET treated and untreated samples in toluene exhibited higher fluorescence yield $\left(\Phi_{\mathrm{f}}=0.14 \pm 0.1\right)$ than observed in aqueous solution $\left(\Phi_{\mathrm{f}}=0.08\right)$. The excited state properties of Au-Gltx in toluene are summarized in Table 1. Since both TOAB and PET treated samples show similar excited state properties, we will discuss the excited state characterization of TOAB capped Au-Glx samples in toluene in the foregoing discussion. 
Table 1. Excited state properties of TOAB capped and PET capped gold nanoclusters.

\begin{tabular}{lcc}
\hline Photophysical properties & TOAB capped AuNCs & PET-treated AuNCs \\
\hline$\Phi_{\mathrm{T}}($ Triplet Yield $)$ & 0.15 & 0.15 \\
$\Phi\left({ }^{1} \mathrm{O}_{2}\right)$ & $0.13 \pm 0.01$ & - \\
$\mathrm{k}_{\mathrm{nr}}$ & $2.40 \times 10^{4} \mathrm{~s}^{-1}$ & $5.60 \times 10^{5} \mathrm{~s}^{-1}$ \\
$\mathrm{k}_{\mathrm{r}}$ & $3.20 \times 10^{5} \mathrm{~s}^{-1}$ & $1.00 \times 10^{5} \mathrm{~s}^{-1}$ \\
$\mathrm{k}_{\mathrm{q}}\left(\mathrm{O}_{2}\right)$ & $7.20 \times 10^{8} \mathrm{M}^{-1} \mathrm{~s}^{-1}$ & $4.00 \times 10^{9} \mathrm{M}^{-1} \mathrm{~s}^{-1}$ \\
$\mathrm{k}_{\mathrm{et}}(\beta$-Carotene $)$ & $1.00 \times 10^{9} \mathrm{M}^{-1} \mathrm{~s}^{-1}$ & - \\
\hline
\end{tabular}
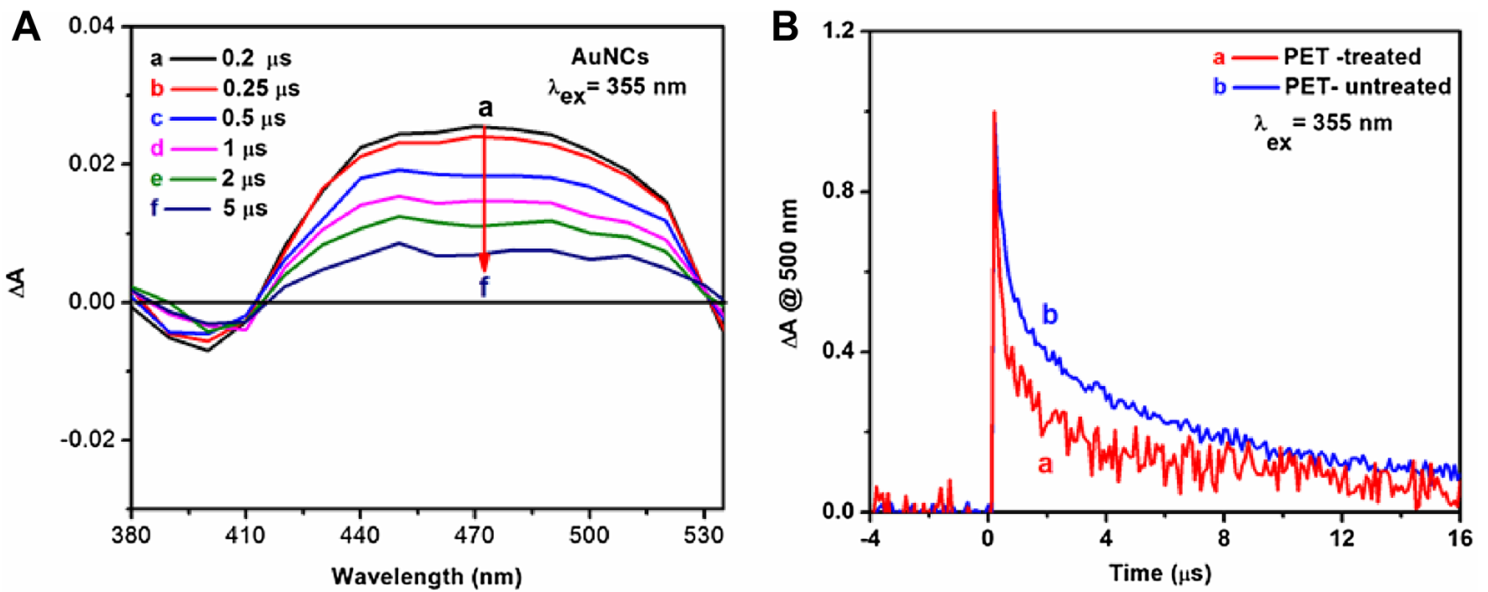

Figure 3. (A) Triplet-Triplet absorption spectra of TOAB capped Au-Glx clusters in deaerated toluene (Ex $355 \mathrm{~nm}$ laser pulse). (B) Absorption decay traces monitored at $500 \mathrm{~nm}$ following $355 \mathrm{~nm}$ laser pulse excitation of AuNCs treated with 2-phenylethanethiol (a, red) and AuNCs in toluene (b, blue).

\subsection{Triplet excited state characterization using nanosecond laser flash photolysis}

The gold clusters exhibit relatively long-lived triplet excited state. As shown in the previous study, the relatively long-lived excited state arises from the ligand to charge transfer state between glutathione shell and Au core. ${ }^{3,12}$ In the present study, we subjected the gold cluster suspension to $355 \mathrm{~nm}$ laser pulse excitation and transient behavior was recorded at different visible wavelengths. The time-resolved difference absorption spectra recorded after $355 \mathrm{~nm}$ laser pulse excitation is shown in Figure 3A. The difference absorption spectrum shows broad absorption in the visible $(415-530 \mathrm{~nm})$ region. Figure 3B shows the decay of the triplet excited state. The lifetimes measured for TOAB capped and PET capped gold clusters were 3.7 and $1.5 \mu \mathrm{s}$, respectively. The excited state characteristics of PET capped AuNCs also exhibit similar characteristics (Figure S1 in Supplementary Information.) These lifetimes are significantly longer than the one measured for Au-Glx clusters in water. ${ }^{12}$ The additional protection provided by the organic capping ligand is expected to provide rigidity for the excited state. The increased luminescence, as well as increased excited state lifetime, make these systems kinetically more prudent to participate in energy and electron transfer processes. The insensitivity to surface capped molecules (viz., TOAB and PET) to the excited state lifetime shows its indifference to the additional capping of the organic layer. These studies further show the versatility of the present approach to tune the capping of gold clusters without significantly affecting the excited state properties.

\subsection{Energy transfer to $\beta$-carotene}

The excited state properties of TOAB capped gold clusters were further probed with Triplet-Triplet (T-T) energy transfer to $\beta$-carotene. The transient absorption spectra recorded in Figure 4A shows the evolution of the triplet $\beta$-carotene excited state following the excitation of AuNCs in toluene (reactions 1 and 2). $\beta$-Carotene with its triplet energy of $19.4 \pm 1.0 \mathrm{kcal} \mathrm{mol}^{-1}$ has been extensively used as an acceptor to probe T-T energy transfer of the triplet excited state. ${ }^{18,19}$ 

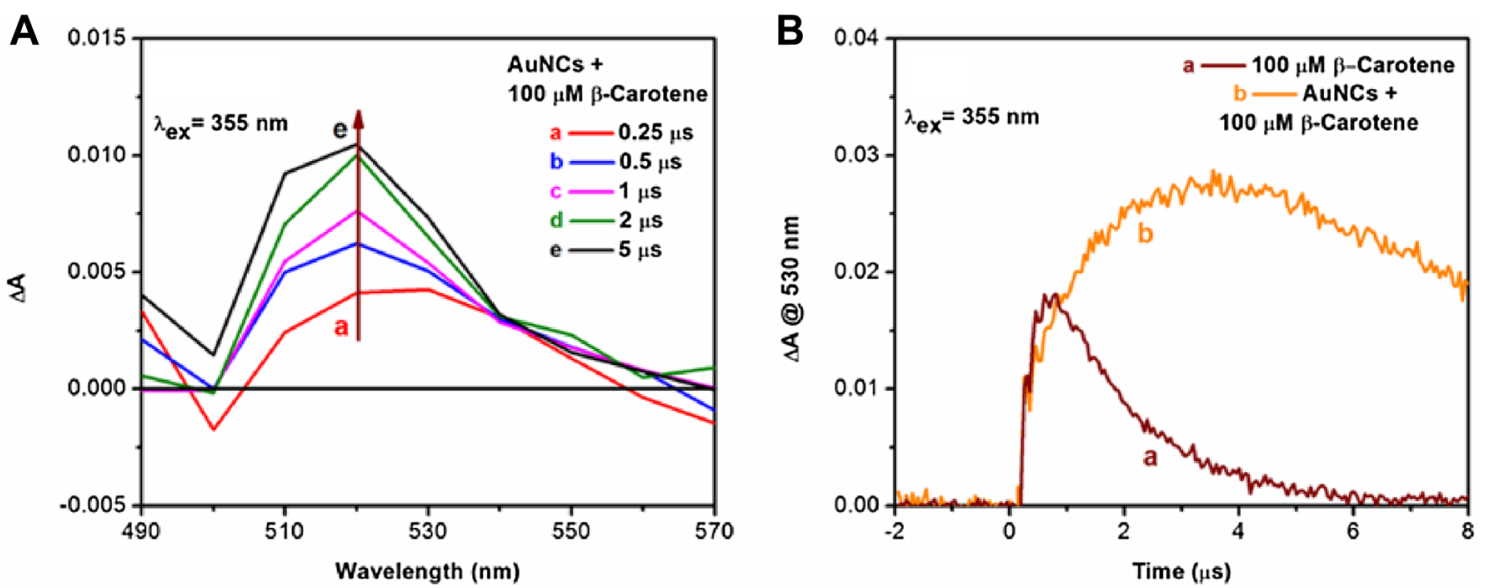

Figure 4. (A) Transient absorption spectra recorded in a nanosecond laser flash photolysis experiment. AuNCs solution in toluene (deaerated) containing $100 \mu \mathrm{M}$ of $\beta$-Carotene were excited with $355 \mathrm{~nm}$ laser pulse excitation. (B) Absorption kinetic decay traces of (a) $100 \mu \mathrm{M} \beta$-Carotene in toluene and (b) $100 \mu \mathrm{M}$ of $\beta$-Carotene with AuNCs in toluene monitored at $520 \mathrm{~nm}$.

$\mathrm{AuNC}+\mathrm{h} \nu \rightarrow \mathrm{AuNC}^{*}$

AuNC $^{*}+\beta$-Carotene $\rightarrow$ AuNC $+\beta$-Carotene*

The T-T energy transfer is also realized from the growth of the absorption recorded at $530 \mathrm{~nm}$. The initial quick rise is due to the direct excitation of $\beta$-carotene which can also be seen when $\beta$-carotene is excited directly with $355 \mathrm{~nm}$ laser pulse. On the other hand, when AuNCs are excited in the presence of $\beta$-carotene we see the formation of $\beta$-carotene triplet excited state. The growth of the absorption at $530 \mathrm{~nm}$ confirms the ability of AuNCs to transfer energy to lower lying triplet of $\beta$-carotene. Additional experimental laser flash photolysis results are presented in Figure S2 in the Supplementary Information.

Various photophysical properties including quenching rate constants obtained from the laser flash photolysis experiments are summarized in Table 1.

\subsection{Singlet oxygen generation}

Since the triplet excited state is energetic enough to induce T-T energy transfer we explored the possibility of formation of singlet oxygen generation. Triplet energy transfer from excited dye to ground state oxygen has been commonly employed to generate singlet oxygen $\left(\mathrm{E}\left({ }^{1} \mathrm{O}_{2}\right), 22.6 \mathrm{kcal} \mathrm{mol}^{-1}\right) .{ }^{18}$ Figure 5 shows the emission spectra of AuNCs in a toluene solution containing different concentrations of oxygen. A gas mixture of $\mathrm{O}_{2}: \mathrm{N}_{2}$ of different ratios were used to purge the solution and achieve desired oxygen concentration. The Stern-Volmer quenching analysis for TOAB capped and PET capped AuNCs are presented in Figures S3 and S4
(Supplementary Information). The decreased emission with increasing concentration confirms the ability of oxygen to quench the excited state of AuNCs.

$\mathrm{AuNC}^{*}+{ }^{3} \mathrm{O}_{2} \rightarrow \mathrm{AuNC}+{ }^{1} \mathrm{O}_{2}^{*}$

We also employed nanosecond laser flash photolysis to estimate the quenching rate constant of reaction 3 . The observed pseudo first-order rate constant versus oxygen concentration is plotted in Figure 5B. From the slope of the straight line plot, we obtain a bimolecular rate constant of $7.2 \times 10^{8} \mathrm{M}^{-1} \mathrm{~s}^{-1}$. Similarly obtained oxygen quenching rate constant for PET-treated AuNCs was $4 \times 10^{8} \mathrm{M}^{-1} \mathrm{~s}^{-1}$.

In order to further quantify the singlet oxygen formation in AuNCs suspension, we carried out photolysis experiments in the presence of 1,3-Diphenylisobenzofuran (DPBF). This molecule undergoes rapid 1,4-cycloaddition to produce colorless endoperoxides (1,2-dibenzoylbenzene). The irreversibility of this photochemical reaction (reaction 4) results in the disappearance of the visible absorption band and thus indirectly allows one to estimate the singlet oxygen production quantitatively. ${ }^{20}$

$$
\begin{aligned}
& \text { DPBF }+{ }^{1} \mathrm{O}_{2} \\
& \quad \rightarrow \text { Oxidation Product (1, 2-dibenzoylbenzene) }
\end{aligned}
$$

The formation of singlet oxygen by irradiation of gold nanoparticles in their plasmon resonance band with continuous or pulsed laser light has been investigated. ${ }^{21}$ Figure 6(A) shows the absorption spectra of DPBF and AuNC suspension in toluene (air saturated) following the irradiation with $470 \mathrm{~nm}$ light irradiation. With 

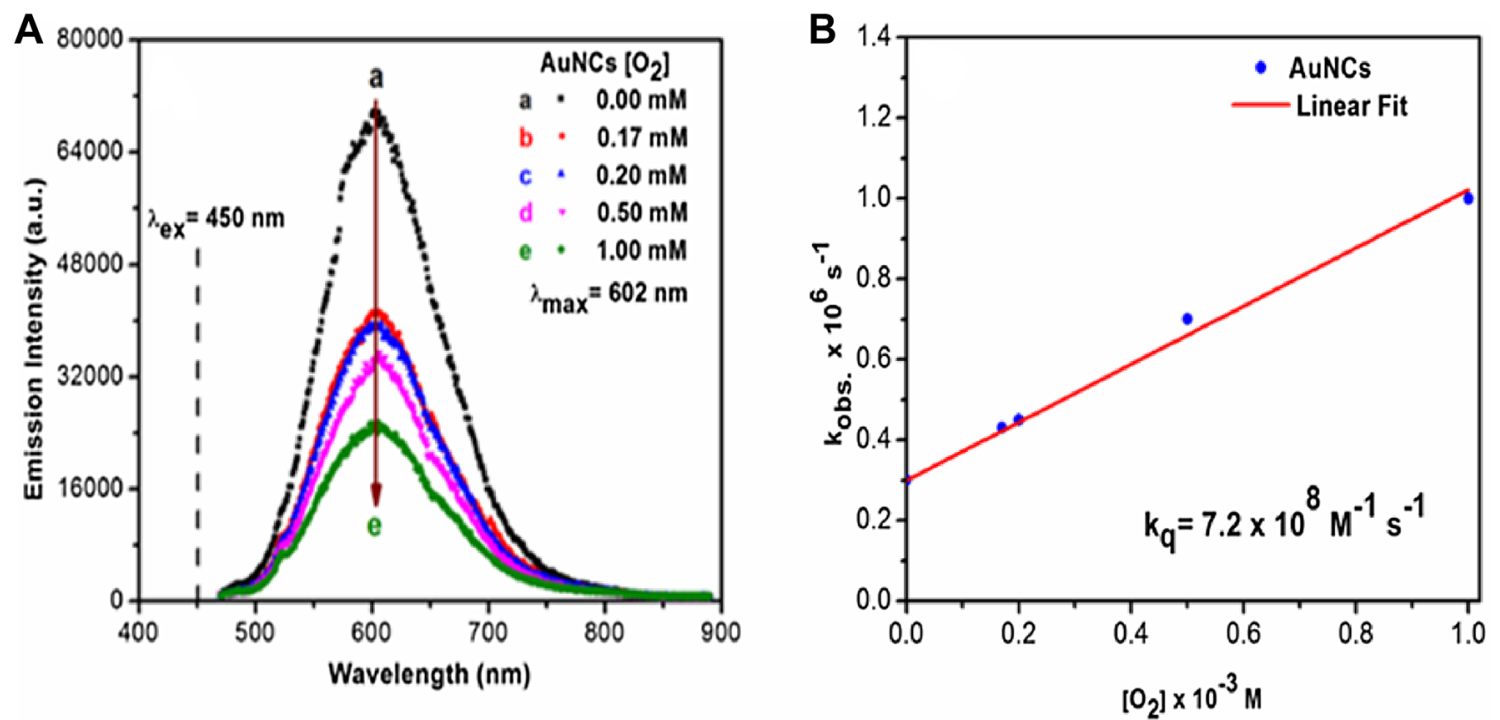

Figure 5. (A) Emission $\left(\lambda_{\mathrm{ex}}: 450 \mathrm{~nm}\right)$ spectra of PET-untreated AuNCs in toluene with varying concentrations of oxygen ranging from $0 \mathrm{mM}$ to $1 \mathrm{mM}$ (a-e). (B) Fit of observed rate constant of excited state (untreated AuNCs in toluene) decay from nanosecond laser flash photolysis versus concentrations of molecular oxygen. A bimolecular rate constant of $7.2 \times 10^{8} \mathrm{M}^{-1} \mathrm{~s}^{-1}$ was obtained for oxygen quenching reaction.

A

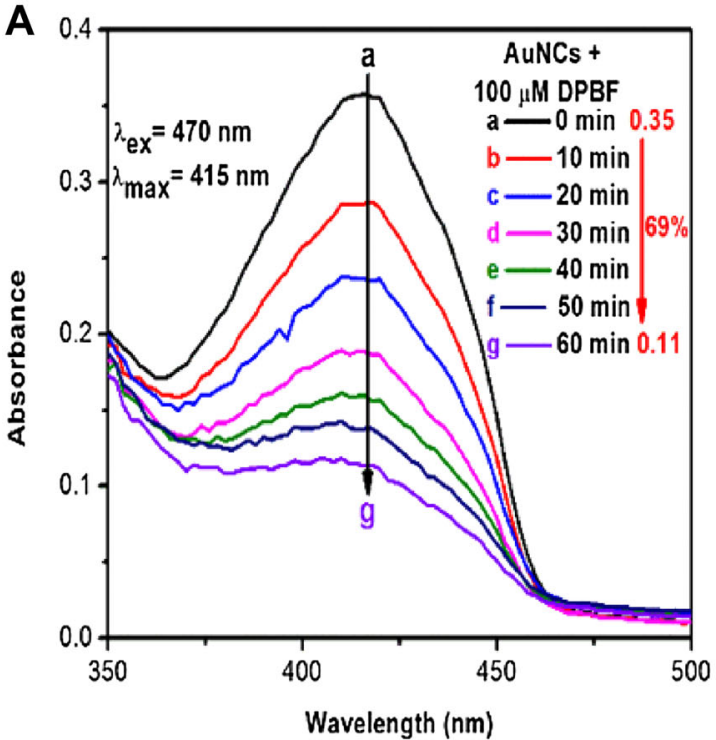

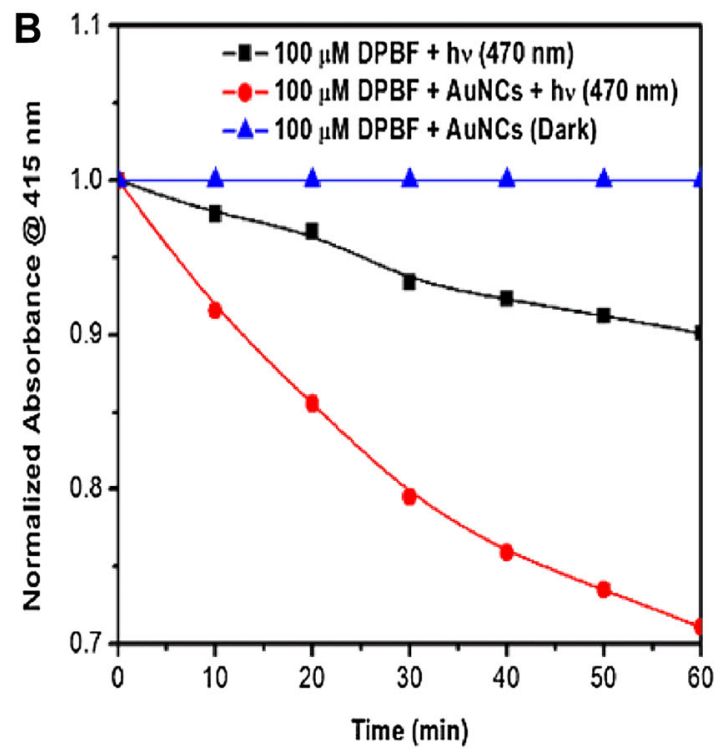

Figure 6. (A) Absorption spectra of PET-untreated AuNCs in toluene with $100 \mu \mathrm{M}$ of 1,3-Diphenylisobenzofuran (DPBF) illuminated under $470 \mathrm{~nm}$ light for $1 \mathrm{~h}$, spectra recorded at intervals of $10 \mathrm{~min}$. (B) Time versus absorbance plot of (a) $100 \mu \mathrm{M}$ DPBF and (b) AuNCs with $100 \mu \mathrm{M}$ DPBF with $470 \mathrm{~nm}$ excitation.

(c) The control experiment for (b) with no excitation.

increasing time we see a decrease in the absorbance of DPBF band (abs. max. $415 \mathrm{~nm}$ ). Figure 6B shows the decrease in absorbance at $415 \mathrm{~nm}$ following the excitation of DPBF solution with and without AuNCs. DPBF alone undergoes slow degradation if excited with visible light. However, the decay becomes rapid in the presence of AuNCs. This further confirms the participation of AuNCs in the overall degradation of DPBF.
The formation of singlet oxygen in irradiated AuNC suspension as confirmed from the disappearance of the DPBF absorption was further evaluated for the quantum yield. We repeated the same experiment with Anthracene as the photosensitizer (Figure S5, Supplementary Information). By comparing the yield of disappeared absorption of DPBF we estimate the quantum yield of singlet oxygen generation in AuNC 
system to be 0.13 . The similarity between the quantum yields of triplet excited state of AuNC and singlet oxygenation shows that the gold clusters are very efficient in transferring energy to ground state oxygen. Given the importance of singlet oxygen in photodynamic therapy, it is important to recognize the potential application of gold clusters in photodynamic therapy.

\section{Conclusions}

To summarize, we have successfully transferred AuNCs in an organic solvent through capping with TOAB or PET. The versatility of capping with organic molecules opens up new ways to use these AuNCs in photochemical and photobiological applications. The ability of excited NCs to produce singlet oxygen is another interesting property that has been explored for the first time.

\section{Supplementary Information (SI)}

Experimental procedures and additional nanosecond laser flash photolysis results are presented in the Supplementary Information. This information is available at www.ias.ac.in/ chemsci.

\section{Acknowledgements}

The research work described here was supported by the Division of Chemical Sciences, Geosciences, and Biosciences, Office of Basic Energy Sciences of the U.S. Department of Energy, through award DE-FC02-04ER15533. This is contribution number NDRL No. 5218 from the Notre Dame Radiation Laboratory.

\section{References}

1. Li G and Jin R 2013 Atomically precise gold nanoclusters as new model catalysts Acc. Chem. Res. 461749

2. Abbas M A, Kamat P V and Bang J H 2018 Thiolated gold nanoclusters for light energy conversion ACS Energy Lett. 3840

3. Stamplecoskie K G, Chen Y-S and Kamat P V 2014 Excited-state behavior of luminescent glutathioneprotected gold clusters J. Phys. Chem. C 1181370

4. Ohta T, Shibuta M, Tsunoyama H, Negishi Y, Eguchi $\mathrm{T}$ and Nakajima A 2013 Size and structure dependence of electronic states in thiolate-protected gold nanoclusters of Au25(SR)18, Au38(SR)24, and Au144(SR)60 J. Phys. Chem. C 1173674

5. Qian H, Zhu M, Wu Z and Jin R 2012 Quantum sized gold nanoclusters with atomic precision Acc. Chem. Res. 451470
6. Jin R, Qian H, Wu Z, Zhu Y, Zhu M, Mohanty A and Garg N 2010 Size focusing: A methodology for synthesizing atomically precise gold nanoclusters J. Phys. Chem. Lett. 12903

7. Zhu M, Qian H and Jin R 2010 Thiolate-protected $\mathrm{Au}_{24}\left(\mathrm{SC}_{2} \mathrm{H}_{4} \mathrm{Ph}\right)_{20}$ nanoclusters: Superatoms or not? $J$. Phys. Chem. Lett. 11003

8. Chen Y-S, Choi H and Kamat P V 2013 Metal cluster sensitized solar cells. A new class of thiolated gold sensitizers delivering efficiency greater than $2 \% \mathrm{~J}$. Am. Chem. Soc. 1358822

9. Chen Y-S and Kamat P V 2014 Glutathione capped gold nanoclusters as photosensitizers. Visible light induced hydrogen generation in neutral water J. Am. Chem. Soc. 1366075

10. Choi H, Chen Y-S, Stamplecoskie K G and Kamat P V 2015 Boosting the photovoltage of dye-sensitized solar cells with thiolated gold nanoclusters J. Phys. Chem. Lett. 6217

11. Stamplecoskie K G and Kamat P V 2015 Synergistic Effects in the Coupling of Plasmon Resonance of Metal Nanoparticles with Excited Gold Clusters J. Phys. Chem. Lett. 1870

12. Stamplecoskie K G and Kamat P V 2014 Size-dependent excited state behavior of glutathione-capped gold clusters and their light-harvesting capacity J. Am. Chem. Soc. 13611093

13. Yu $\mathrm{Y}$, Chen $\mathrm{X}$, Yao $\mathrm{Q}, \mathrm{Yu} \mathrm{Y}$, Yan $\mathrm{N}$ and $\mathrm{Xie}$ J 2013 Scalable and Precise Synthesis of Thiolated $\mathrm{Au}_{10-12}, \mathrm{Au}_{15}, \mathrm{Au}_{18}$, and $\mathrm{Au}_{25}$ Nanoclusters via $\mathrm{pH}$ Controlled CO Reduction Chem. Mater. 25946

14. Brust M, Walker M, Bethell D, Schffrin D J and Whyman R 1994 Synthesis of thiol-derivatized gold nanoparticles in a two-phase liquid-liquid system Chem. Commun. 801

15. Brust M, Fink J, Bethell D, Schiffrin D J and Kiely C 1995 Synthesis and reactions of functionalized gold nanoparticles J. Chem. Soc. Chem. Comm. 1655

16. Thomas K G, Zajicek J and Kamat P V 2002 Surface binding properties of tetraoctylammomium bromide capped gold nanoparticles Langmuir 183722

17. Luo Z T, Yuan X, Yu Y, Zhang Q B, Leong D T, Lee J $Y$ and Xie J P 2012 From aggregation-induced emission of $\mathrm{Au}(\mathrm{I})$-thiolate complexes to ultrabright $\mathrm{Au}(0) @ \mathrm{Au}(\mathrm{I})$ thiolate core-shell nanoclusters J. Am. Chem. Soc. 134 16662

18. Lambert C and Redmond R W 1994 Triplet energy level of $\beta$-carotene Chem. Phys. Lett. 228495

19. Nielsen B R, Jørgensen K and Skibsted L H 1998 Triplettriplet extinction coefficients, rate constants of triplet decay and rate constant of anthracene triplet sensitization by laser flash photolysis of astaxanthin, $\beta$-carotene, canthaxanthin and zeaxanthin in deaerated toluene at $298 \mathrm{~K}$ J. Photochem. Photobiol. A: Chem. 112127

20. Zhang X-F and Li X 2011 The photostability and fluorescence properties of diphenylisobenzofuran J. Lumin. 1312263

21. Chadwick S J, Salah D, Livesey P M, Brust M and Volk M 2016 Singlet oxygen generation by laser irradiation of gold nanoparticles J. Phys. Chem. C 12010647 